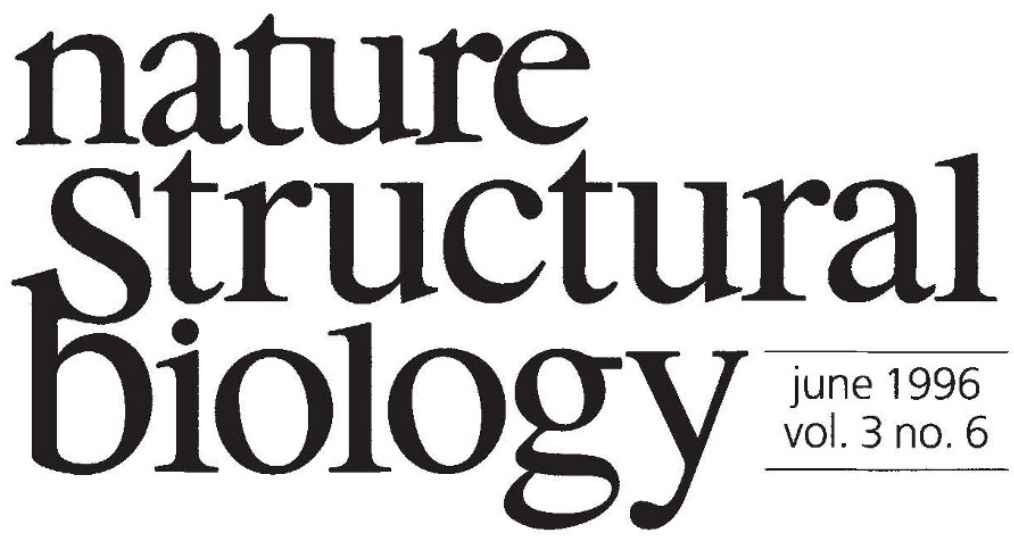

\title{
How to really heal with crystals
}

The more basic the biological problem, the more removed it tends to be from practical (read: medical) application. This truism is countered by the difficult-tosubstantiate idea that medicine never knows from where its next big advance will come. This has always been the public defence of basic researchers when they are called on to justify their own 'curiosity driven' science. Sometimes, though, the divide between curiosity and application is nonexistant: a clear illustration of the interdependency of basic research and medicine can be seen in the community of scientists working on the serine proteinase inhibitors (serpins), who met for a recent symposium*.

There can be no doubt about the practical interest of the serpins, which regulate blood coagulation and fibrinolysis, blood pressure, inflammation and cell death, cell migration and attachment, and play a critical part in development (including a role in insect ecdysis). This means that they have the potential as remedies for thrombosis, atherosclerosis, trauma, stroke, cancer, and the ravages of insect pests-a list of modern plagues that would have made Pharaoh himself relent.

This potential was seen in presentations at the symposium. Mice lacking a functional gene for plasminogen activator inhibitor-1 (PAI-1) have a bleeding disorder similar to one identified in humans also deficient in PAI-1; conversely, overexpression of PAI-1 leads to drastically increased susceptibility to atherosclerotic plaques, probably by inhibiting the degradation of fibrin (D.Ginsburg, University of Michigan). A serpin called maspin, downregulated in breast carcinoma, prevents cells in vitro from migrating and invading extracellular matrix (R. Sager, Dana Farber Cancer Institute, Boston). Specific serpin variants, injected into animals, protect against inflammation - up to $80 \%$ of pre-treated rats survived an NIH-approved test for trauma that usually kills all of them (H. Rubin, University of Pennsylvania, Philadelphia).

With this potential, it is no wonder that serpins are a subject for structural research. The necessity of a basic understanding is underscored by the fact that serpins tend not to maintain their specificity across species, so animal models are only indirectly applicable to human studies. There is no choice but to try to understand their mechanism. But serpins are an interesting class of molecules in

*International symposium on the Chemistry and Biology of Serpins. University of North Carolina at Chapel Hill, April 13-16 1996. their own right, and even if there were no medical imperative, structural biologists would be drawn to them on the basis of curiosity alone.

The primary basic interest of serpins lies in the fact that they are metastable proteins. (Proteins are classed as serpins based on sequence homologies. This discussion applies to the inhibitory members of the class.) The 'business end' of an inhibitory serpin is its reactive-centre loop (RCL). If the RCL is cleaved, it then 
incorporates into the serpin $\beta$-sheet A. Some serpins can also exist in a latent form, in which loop insertion occurs without cleavage. This considerable structural rearrangement prompts many questions. How does a protein fold into a metastable native state? How is a metastable state produced and maintained (see the study by Lee, Park and $\mathrm{Yu}^{1}$ on page 497 of this issue, which identifies mutations in the core of $\alpha_{1}$-antitrypsin that stabilize the native state). What role does metastability play in the mechanism of inhibition? This last is clearly an important question-even someone unfamiliar with the field could tell, as few of the symposium attendees seemed to be able to agree on a precise answer.

Several variations were presented to describe the general pathway of serpin mechanism. There is agreement that a reversible initial complex between proteinase and inhibitor is formed. Next, the pathway bifurcates: the serpin can be cleaved and released (like a normal substrate); or it can form a tight inhibitory complex-but there is disagreement about whether this last step is reversible. From the inhibitory complex, the serpin (cleaved and inactive) can also be released, but at a relatively slow rate.

The disagreements about the pathway stem largely from two different types of model of how serpins inhibit proteinases. At one simplified extreme, it is possible that serpins act like more standard inhibitors, binding tightly to the proteinase and blocking access to the catalytic triad of the enzyme. In this case, it is hard to relate the serpin's metastability to its' inhibitory activity. At the other extreme, when the serpin binds to inhibitor, the RCL is cleaved and a covalent acyl enzyme (but it may be tetrahedral) intermediate is formed. RCL insertion (which may occur to varying degrees) is coupled to these events, putting strain on the proteinase activesite serine, distorting the catalytic triad. Cumulative evidence favours something closer to the second model: for example, mutations at the hinge residues of the RCL, which presumably inhibit loop insertion, result in more substrate-like behaviour. Apparently, inhibiting insertion may allow the proteinase more opportunity to cleave and inactivate the serpin; there is also evidence suggesting that the RCL is cleaved in the complex (D. Lawrence, American Red Cross Holland Laboratory, Bethesda). Further support comes from NMR results indicating that in the complex, the proteinase catalytic triad is distorted (H. Rubin). However, the standard mechanism, although perhaps nailed to its perch, is not dead yet. For example, standard mechanism inhibitors, which do not require cleavage or covalent intermediates, can form SDS-stable complexes with their targets (T. Komiyama, Duke University, Durham). Since SDS stability has been taken as evidence for covalent complex formation in serpin-proteinase interaction, more work is required to unambiguously establish the formation of a covalent intermediate in serpins. The reversibility some observe in the formation of the inhibitory complex is also difficult to reconcile with RCL cleavage. It will be interesting to see whether the core mutations that stabilize a serpin ${ }^{1}$ also affect partitioning, as do the hinge mutations. But all-in-all, the non-standard mechanism is more attractive, if only because it is otherwise hard to imagine why metastability is preserved in so many serpins.

A crystal structure of a complex would probably put much of this dispute to rest, but technical difficulties in crystallizing a complex may mean that less direct evidence must do for the near future. Even a result shedding light on the slow release of cleaved serpin from the inhibitory complex could have implications for designing better inhibitors. In the meantime, the best course may be for the community to meet regularly, if only to agree on what questions it has yet to answer. And unanswered questions are not always a bad thing, particularly when they draw the curious into an important practical problem.

1. Lee, K.N., Park, A.D. \& Yu, M.-H. Nature Struct. Biol. 3, 497-500 (1996). 